\title{
Dr. Jadon replies
}

To the Editor:

We thank Dr. Danve ${ }^{1}$ for taking the time to read our systematic review titled "Serum Soluble Bone Turnover Biomarkers in Psoriatic Arthritis and Psoriatic Spondyloarthropathy"2. The primary objective of this systematic review was to identify serum biomarkers of psoriatic arthritis (PsA) and/or psoriatic spondyloarthritis (PsSpA). Our search criteria, therefore, cast a net wide enough to find all such literature. We did not find any literature on serum biomarkers in PsSpA, which is an important finding in itself and has/will inform future research in the PsA research community. It is important, therefore, to include "PsSpA" in the title of the paper to ensure that any reader or researcher wishing to find a review on this topic can do so easily. We therefore stand by our decision to include "PsSpA" in the paper title, and do not feel that the title is misleading.

DEEPAK JADON, MBBCh, MRCP, Rheumatology Research Fellow, Royal National Hospital for Rheumatic Diseases, Rheumatology, Upper Borough Walls, Bath, Somerset, BA1 1RL, UK. Address correspondence to Dr. D.

Jadon, e-mail: jadondr@yahoo.com

\section{REFERENCES}

1. Danve A. Is the title appropriate? J Rheumatol 2015;42:1726.

2. Jadon DR, Nightingale AL, McHugh NJ, Lindsay MA, Korendowych E, Sengupta R. Serum soluble bone turnover biomarkers in psoriatic arthritis and psoriatic spondyloarthropathy. J Rheumatol 2015;42:21-30.

J Rheumatol 2015;42:9; doi:10.3899/jrheum.150589 